\title{
Patients choose certainty over burden in bladder cancer surveillance
}

\author{
Frits H. M. van Osch ${ }^{1,2}$. Duncan Nekeman ${ }^{3}$ - Neil K. Aaronson ${ }^{4}$. Lucinda J. Billingham ${ }^{5}$. Nicholas D. James ${ }^{1}$. \\ K. K. Cheng ${ }^{3} \cdot$ Richard T. Bryan ${ }^{1} \cdot$ Maurice P. Zeegers ${ }^{6}$
}

Received: 2 January 2019 / Accepted: 11 March 2019 / Published online: 23 March 2019

(c) The Author(s) 2019

\begin{abstract}
Background Due to the high risk of recurrence of non-muscle invasive bladder cancer, all patients undergo regular cystoscopic surveillance for early detection. As cystoscopy is invasive, costly and increases the burden of the disease considerably, there is significant ongoing research and development into non-invasive urinary biomarker substitutes. This study aims to assess the level of sensitivity required before patients accept a new urinary biomarker.

Methods We studied the preferences for a hypothetical diagnostic urinary biomarker and compared this to usual care (cystoscopy) at different levels of sensitivity among 437 patients with bladder cancer (354 men and 83 women) from the UK Bladder Cancer Prognosis Programme. A standard gamble approach was used to estimate the minimally acceptable sensitivity (MAS) of the new biomarker. Additionally, non-parametric statistical analyses were performed to investigate the association between surveillance preference and various patient characteristics.

Results Almost half of patients $(183,43 \%)$ would not replace cystoscopy with a urinary biomarker unless it was $100 \%$ sensitive. The median MAS was $99.9999 \%$, and nearly $85 \%$ of patients demanded a sensitivity of at least $99 \%$ before preferring a urinary biomarker test over cystoscopy. These results were consistent across all patient characteristics and demographic categories.

Conclusions Our results indicate that patients demand urinary biomarkers as sensitive as cystoscopy before they would be willing to forego cystoscopy for bladder cancer surveillance.
\end{abstract}

Keywords Non-muscle-invasive bladder cancer $\cdot$ Non-invasive biomarkers $\cdot$ Sensitivity and specificity $\cdot$ Standard gamble

Richard T. Bryan and Maurice P. Zeegers contributed equally.

Frits H. M. van Osch

f.vanosch@maastrichtuniversity.nl

1 Institute of Cancer and Genomic Sciences, University of Birmingham, Birmingham, UK

2 Department of Complex Genetics, Nutrition and Translational Research in Metabolism (School NUTRIM), Maastricht University, PO Box 616, 6200 MD Maastricht, The Netherlands

3 Department of Public Health, Epidemiology and Biostatistics, School of Health and Population Sciences, University of Birmingham, Birmingham, UK

4 Division of Psychosocial Research and Epidemiology, The Netherlands Cancer Institute, Amsterdam, The Netherlands

5 Cancer Research UK Clinical Trials Unit, University of Birmingham, Birmingham, UK

6 Department of Complex Genetics, Nutrition and Metabolism in Translational Research (NUTRIM), Care and Public Health Research Institute (CAPHRI), Maastricht University, Maastricht, The Netherlands

\section{Introduction}

Bladder cancer is the ninth most common malignancy worldwide, with a rising global incidence [1]. The majority of patients (75-80\%) present with non-muscle-invasive disease (NMIBC) [2,3]. Although not immediately life threatening in the majority of cases, recurrence and progression of NMIBC remain significant issues [4, 5], with up to 55\% of patients experiencing recurrence within 5 years of diagnosis [6]. Current guidelines recommend long-term surveillance except for low-risk NMIBC after 12 months [5]. With a global prevalence that can be estimated at over 2,000,000, at any one time, there will be a very considerable number of patients requiring such surveillance [1].

Surveillance typically comprises outpatient flexible cystoscopy and urine cytology [5, 7]. For patients with low-risk NMIBC, European Association of Urology (EAU) guidelines recommend follow-up cystoscopy and urine cytology at 3 months and 12 months after tumour resection (TURBT), 
and then annually for the next 5 years. Patients with highrisk NMIBC undergo more intensive surveillance-every 3 months for the first 2 years, every 6 months until 5 years, and yearly thereafter, most likely for the rest of their lives [5]. If recurrence is detected, then the tumour is resected and surveillance will re-commence from the beginning. It has been estimated that each cystoscopy and urine cytology episode costs $£ 533$ [8]; consequently, bladder cancer is the most expensive cancer to treat on a per patient basis from diagnosis to death, with the majority of expense attributable to NMIBC $[9,10]$.

Cystoscopy itself significantly increases the burden of disease-it is an invasive procedure that causes pain and discomfort in about one-third of patients [11]. In contrast, previous studies have shown that the only burden attributable to a non-invasive test (such as a urinary biomarker) is the waiting time for the test result [11]. For these reasons, a number of diagnostic urinary biomarkers have been developed by academia and industry in an attempt to create less burdensome and less costly NMIBC surveillance regimens.

Urine cytology is widely used as an adjunct to cystoscopy. It has high sensitivity for detecting high-grade disease $( \pm 80 \%)$, but poor sensitivity for low-grade disease $( \pm 30 \%)$ $[12,13]$; in addition, diagnostic accuracy depends upon a number of confounding factors, such as the quality of the sample and the level of expertise of the cytopathologist [14].

More recently, other types of diagnostic urinary biomarkers have been developed, including soluble urine markers and exfoliated cell markers, as well as multigene urinary DNA-based tests [15, 16]; some are commercially available and US FDA approved [17]. As summarised by Soria et al. in 2018, such biomarkers have moderate to good sensitivity, and multigene panels can reach sensitivity levels of over $90 \%$ in patients with high-grade tumours [17]. Biomarkerdriven surveillance might become a realistic possibility if, or when, they reach a sufficient and consistent level of sensitivity [18], bearing in mind that cystoscopy itself is operator dependent with the sensitivity and specificity of conventional white light cystoscopy estimated to be up to $85 \%$ and $87 \%$, respectively $[9,19]$.

However, what is less clear is what level of sensitivity is acceptable to patients undergoing surveillance for NMIBC, such that they would be willing to switch from cystoscopy, which in practitioners' eyes is perceived as the golden standard. Only two studies have previously published on this subject. Vriesema et al., in a utility analysis on 85 patients (70 men and 15 women) undergoing bladder cancer surveillance in The Netherlands, found that $68 \%$ of their patients had a minimally acceptable sensitivity (MAS) of over $99 \%$ [20]. The more cystoscopies a patient had undergone, the higher was their MAS, suggesting that the acceptability of cystoscopy increases with patients' familiarity with and/or confidence in the procedure. In a similar utility analysis on
200 patients (119 men and 81 women), Yossepowitch et al. reported that $70 \%$ of 200 patients recruited in the United States had a MAS over $99 \%$, and only $24.5 \%$ would accept a MAS lower than 95\% [21].

The objective of this study was to assess the level of biomarker acceptability in a NMIBC population naïve to conventional cystoscopic surveillance, as well as assessing factors that may affect levels of acceptability, such as sociodemographics and social support.

\section{Materials and methods}

This study is part of the West Midlands Bladder Cancer Prognosis Programme BCPP), which is an ongoing multicentre patient cohort study in the West Midlands region of the UK [22]. Adult patients (age $>18$ years) presenting with symptoms suspicious for bladder cancer haematuria in over $80 \%$ [23] in the 10 participating urology centres within the region were enrolled on the basis of cystoscopic findings suggestive of bladder cancer. Those who had a previous diagnosis of cancer of the urethra, bladder, ureter or renal pelvis within the last decade, HIV infection, or any other condition that might interfere with the safety of the participant were excluded. The study received ethical approval as part of BCPP (reference: 06/MRE04/65), and written informed consent was obtained from all participants.

At the time of diagnosis, trained research nurses conducted semi-structured face-to-face interviews to collect information on socio-demographics, health-related lifestyle (lifetime smoking history, passive smoking, use of hair dye), medical and drug history, dietary intake, social support and HRQoL. HRQoL was assessed with the European Organisation for Research and Treatment of Cancer EORTC) Quality of Life Questionnaire, the QLQ-C30 [24]. Social support was assessed with the Duke-UNC Functional Social Support Questionnaire FSSQ) [25]. The FSSQ is an eight-item instrument, scored on a 1-5 scale, which measures the strength of a person's social support network. Both the QLQC30 and the FSSQ were converted to a scale ranging from 0 to 100 , with 0 as the lowest quality of life or lowest level of perceived social support and 100 as the highest. Only the overall HRQoL scale from the QLQ-C30 was used, as we hypothesised that the burden of the cystoscopy would affect overall HRQoL rather than any specific HRQOL domain or symptom. Participants were followed up at approximately 3 months post-baseline measurement with a similar questionnaire. During this follow-up, changes in health-related lifestyle and HRQoL were assessed.

We employed a utility-based patient preference questionnaire to assess the patient's preference for cystoscopy versus a hypothetical urinary biomarker test. Using a 'standard gamble' procedure, we posed a series 
of questions in which the patient was asked to choose between two surveillance tests, a cystoscopy or a hypothetical urinary biomarker test [26]. The sensitivity of the hypothetical urinary biomarkers started at $100 \%$ and decreased in each subsequent question. The definition of sensitivity was explained to the patient as the number of tumours missed by the biomarker out of a thousand. The MAS was defined as the lowest value of accuracy at which the biomarker was favoured over the cystoscopy.

Due to the non-normal distribution of the MAS data, we used non-parametric statistical analyses to investigate the association between surveillance preference and various patient characteristics. This included the Mann-Whitney/ Wilcoxon rank-sum test and Spearman's rank correlation coefficient. In addition, we conducted exploratory logistic regression analyses in which we dichotomised the outcome variable (MAS) to identify differences between patients that were willing to sacrifice any sensitivity $(<100 \%)$ and those that were not (100\% only). Stratified analyses were used to identify any possible effect modification. Lastly, sensitivity analyses were conducted where patients with an MAS of "0\%" and an MAS of " $100 \%$ " were excluded.

Fig. 1 Flow chart depicting patient recruitment in the Bladder Cancer Prognosis Programme (BCPP) cohort study and inclusion for the current study
All analyses were performed with Stata/MP version 12. A $p$ value of $<0.05$ was considered statistically significant.

\section{Results}

During the recruitment period (2005-2011), 1536 participants were enrolled. Of these 1536 patients, 326 were excluded because they did not have primary bladder cancer, another 23 were lost to follow-up, and 514 were excluded as they did not complete the first questionnaire, leaving 673 patients potentially available for assessment see Fig. 1. Of these 673 patients, $437(65 \%)$ responded to the standard gamble section and were included in the analyses. These 437 participants were slightly different to the 236 who did not complete the standard gamble with regards to gender $(p=0.05)$ and age $(p=0.04)$, whereby the non-responders were older and more likely to be female (25\% in nonresponders vs. $19 \%$ in responders, $p=0.05$ ).

Of the 437 study participants, 354 were male and 83 were female, the mean age was 69 years old, with a minimum of 33 and maximum of 90 years. 309 patients (73\%) had a partner with whom they were married or living together. The

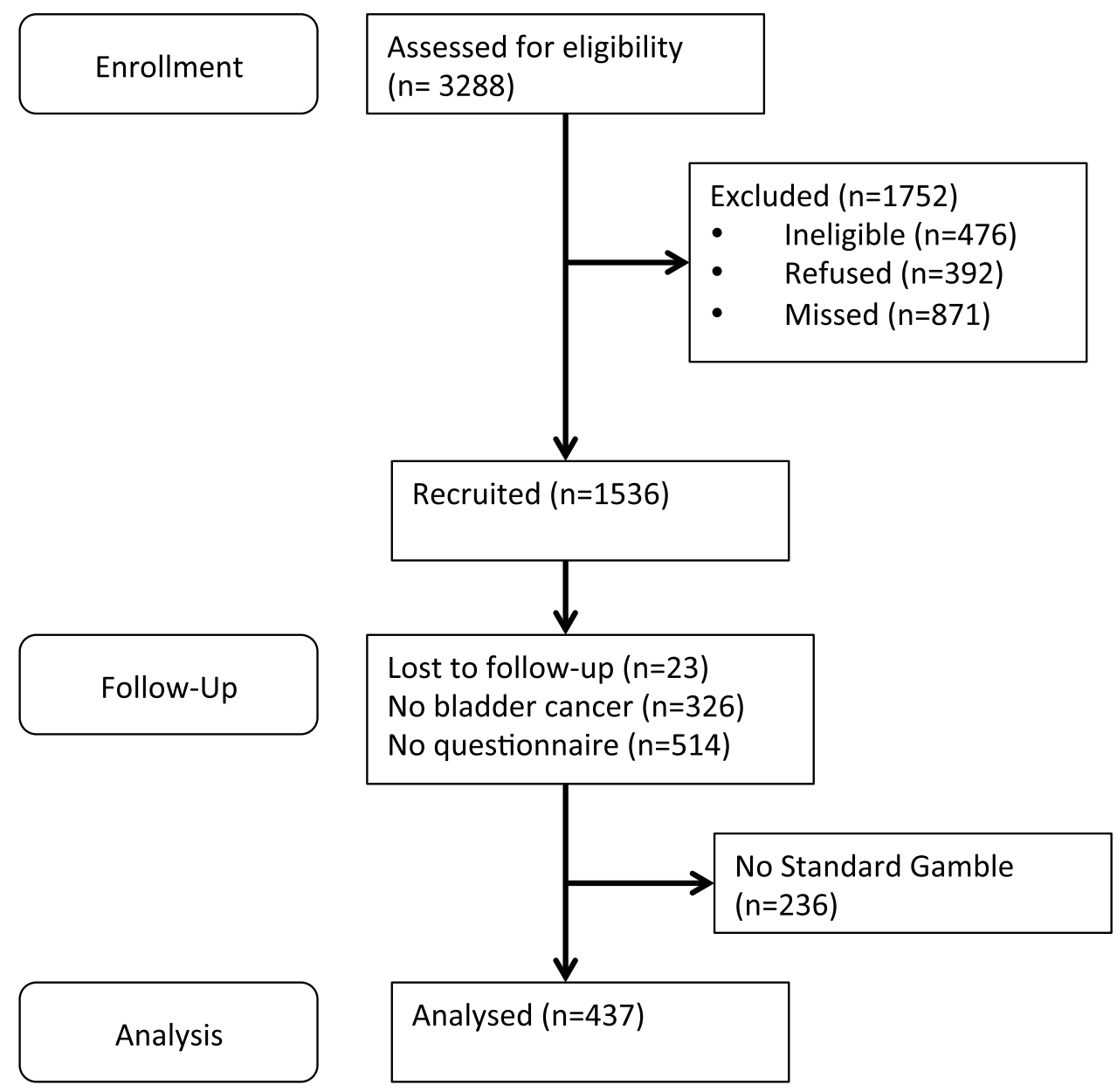


median overall HRQoL as measured by the QLQ-C30 was 75 interquartile range (IQR: 58 -83), and the median social support was 100 (IQR: 88-100) Table 1.

187 patients (43\%) would not change from a cystoscopy to a biomarker unless the biomarker had a sensitivity of $100 \%$. In fact, the median value of MAS was $99.9999 \%$, and nearly $85 \%$ of patients indicated that they would require a sensitivity of at least $99 \%$ before preferring a urinary biomarker test over cystoscopy.

We did not observe any statistically significant associations between the MAS and age $(p=0.092)$, HRQoL $(p=0.161)$, or social support score $(p=0.566)$ Table 2 . The distribution of MAS scores across the categorical variables of gender $(p=0.127)$, marital status $(p=0.374)$ and level of educational attainment $(p=0.060)$ did not differ, and no effect modification was found Table 3.

\section{Discussion}

Our results indicate that the large majority of patients recently diagnosed with bladder cancer demand a very high level of sensitivity before they would be willing to accept urinary biomarkers as an alternative to cystoscopy for periodic surveillance. Based on the conclusions of Vriesema

Table 1 Patient characteristics of all patients included in the analysis

\begin{tabular}{lll}
\hline Age & & \\
Mean (SD) & 68.8 & $(10.4)$ \\
Range & $33-90$ & \\
Sex & & \\
Male (\%) & 348 & $(81 \%)$ \\
Female (\%) & 82 & $(19 \%)$ \\
Marital status & & \\
With partner (\%) & 305 & $(73 \%)$ \\
Without partner (\%) & 111 & $(27 \%)$ \\
General health & & \\
Median (IQR) & 75 & $(58.3-83.3)$ \\
Range & $8-100$ & \\
Social support & & \\
Median (IQR) & 100 & $(87.5-100)$ \\
Range & $0-100$ & \\
Stage & & \\
NMIBC & & $(1 \%)$ \\
pTis (\%) & & \\
pTa (\%) & & \\
pT1 (\%) & 6 & $(57 \%)$ \\
MIBC & 245 & \\
pT2 (\%) & 238 & \\
\hline
\end{tabular}

Non-muscle-invasive bladder cancer (NMIBC)

Muscle-invasive Bladder Cancer (MIBC)
Table 2 Results from Spearman between MAS, age, general health and social support score rank correlation analysis

\begin{tabular}{lc}
\hline Age & \\
Spearman's rho & -0.081 \\
$p$ value & 0.092 \\
General health & \\
Spearman's rho & 0.087 \\
$p$ value & 0.161 \\
Social support score & \\
Spearman's rho & 0.034 \\
$p$ value & 0.566 \\
\hline
\end{tabular}

et al. (2000) [20], we expected that our population of cystoscopy naïve patients would indicate a lower MAS and therefore have a larger spread of responses. However, we observed the opposite. When comparing the results of previous studies to our results, it is clear that patients choose test certainty over test burden.

In all three studies [20,21], more than $65 \%$ of the patients indicated that they would require an MAS of over $99 \%$ and approximately $90 \%$ of patients would require an MAS of over $90 \%$ Table 4 . None of the existing biomarkers are able to consistently achieve this level of sensitivity.

The high percentage of patients that would not prefer a MAS with sensitivity below $100 \%$ may reflect less than a full understanding of the concept of sensitivity, and also the fact that these patients were presented with a hypothetical situation. However, based on qualitative feedback from the research nurses who administered the standard gamble, it is our impression that many patients were "terrified" of the possibility of missing a tumour.

Table 3 Results from Mann-Whitney $U$ and Kruskal-Wallis test comparing rank sums of MAS between sex, marital status and NVQ levels

\begin{tabular}{lcl}
\hline Variable & $n$ per category & Rank sum \\
\hline Sex & 348 & \\
Male & 82 & 76,010 \\
Female & & 19,693 \\
$p$ value of Mann-Whitney $U$ test & 0.127 \\
Marital status & 305 & \\
With partner (\%) & 111 & 66,297 \\
Without partner (\%) & & 22,957 \\
$p$ value of Mann-Whitney $U$ test & & 0.374 \\
National Vocational Qualifications (NVQ) & \\
Level 1 & 43 & 5124 \\
Level 2 & 57 & 7896 \\
Level 3 & 81 & 10,513 \\
Level 4 & 33 & 4513 \\
Level 5 & 37 & 3579 \\
$p$ value of Kruskal-Wallis test & & 0.060 \\
\hline
\end{tabular}


Table 4 MAS distribution in the three different studies

\begin{tabular}{lcccl}
\hline MAS group & $<90$ & 90 to $<99$ & $99-100$ & Total \\
\hline Vriesema et al. & $9(10.6)$ & $18(21.2)$ & $58(68.2)$ & 85 \\
Yossepowitch et al. & $7(3.5)$ & $53(26.5)$ & $140(70.0)$ & 200 \\
Nekeman et al. & $27(6.3)$ & $38(8.8)$ & $365(84.9)$ & 430 \\
Total & $43(6)$ & $109(15)$ & $563(78)$ & 715 \\
\hline
\end{tabular}

It is important to place these findings in the context of conventional white light cystoscopy as the "gold standard" for the diagnosis of NMIBC. However, new optical technologies such as photodynamic diagnosis and narrow band imaging [27] have shown that the sensitivity of conventional white light cystoscopy itself is much less than $100 \%$, and that recurrent tumours are missed in up to $41 \%$ of patients [28]. Most patients in this study will have been unaware of the shortcomings of conventional white light cystoscopy and thus believed and readily accepted the $100 \%$ sensitivity that we used as a cystoscopy benchmark against which biomarkers were to be compared.

We also hypothesised that patients who perceive the burden of the cystoscopy to be high would be more inclined to choose a lower MAS. In a similar manner, patients with a low health-related quality of life (HRQOL) would find the burden of the cystoscopy to be higher and thus choose a lower MAS. In addition, we hypothesised patients with more social support would be willing to take more risk and thus have a lower MAS. Education was taken into account as we hypothesised that patients with a higher education would better understand the risk associated with cystoscopy and the benefit of having a urinary biomarker, even if it comes at the cost of sensitivity. We demonstrated that there was no significant association between MAS and National Vocational Qualifications level, although the highest levels (level four \& five) seemed to report lower MAS values more often $(p=0.060)$.

Even though there was a moderate to high response rate (65\%) in a patient population, we found little evidence of any significant difference between the patients who did and did not take part in the survey. Nevertheless, we cannot rule out the possibility that the MAS of the larger population might be somewhat different from that of our study sample. Furthermore, with bladder-preservation strategies being used more frequently for the treatment of muscle-invasive bladder cancer MIBC) with curative intent [29], one should recognise that this group of patients also require long-term cystoscopic surveillance; their opinions and MAS may differ from those of NMIBC patients. With only a relatively small sample of MIBC patients in this study $(n=37,9 \%)$, we have been unable to make this comparison adjusting for any patient characteristics, although it should be noted that the lowest reported MAS was $90 \%$ in MIBC patients, while
$10.5 \%(n=43)$ of NMIBC patients reported a MAS of $90 \%$ or lower.

Two previous studies by Vriesema et al. and Yossepowitch et al. reported incongruent findings of associations between age and MAS, and gender and MAS; neither study was able to replicate the findings of the other [20,21]. Our study did not replicate findings of either of the two previous studies. This lack of association across the studies between the various potential risk factors and MAS might be due to the small variance in MAS. Additionally, it might be possible that we did not find an association between age and MAS, because mostly older patients did not complete the questionnaire.

Moreover, patients were newly diagnosed when they filled in the questionnaire, increasing the observer-expectancy effect as the subject is in a temporary, vulnerable mind state and the research nurse might show empathy with the patient while a neutral stance is expected. Also, patients were presented with cystoscopy as a $100 \%$ accurate test as their reference, while the actual accuracy of cystoscopy is lower, especially in diagnosing low-grade disease. Future studies should consider rephrasing questions about MAS to make clear that the reference test is not by definition $100 \%$ accurate. Both caveats could increase the risk of inappropriately interpreting results.

Our results suggest that the bladder cancer biomarker research and development community, both commercial and academic, needs to aim for test sensitivity equal to or better than cystoscopy before such tests can be implemented into NMIBC surveillance regimens. This is unlikely to be achievable with the current generation of urinary biomarkers but may be achievable in the near future with urinary DNAbased approaches [16]. Future research should also focus on costs of such non-invasive biomarkers, for example, by investigating how much patients are willing to pay for each increment in diagnostic accuracy. Cystoscopy is unlikely to be replaced today, but tomorrow's urinary biomarkers may allow the frequency of cystoscopic surveillance to be reduced.

In conclusion, our study results indicate that patients demand more sensitive urinary biomarkers than are currently available, thus patients choose certainty over burden.

Acknowledgments With thanks to all participating clinicians and their staff from within the West Midlands region for their continued support: P. Cooke, K. Jefferson, H. Krasnowski, J. Parkin, B.D. Sarmah, P. Ryan, R. Bhatt, M. Foster, K. Desai, L.A. Emtage, K.W. Kadow, C. Luscombe, S. Khwaja, and A. Makar. We would also like to recognise the invaluable contribution made by the BCPP nursing, research and administrative staff to the programme: A. McGuire, C. Langford, C. Letchford, C. Slater, C. Taylor, D. Bird, G. Heritage, H. Shackleford, J. Sears, J. Maiden, J. Goodall, J. Allison, J. Hutton, J.Y. Robinson, K. Castro, L.R. Moore, L. Benson, M. Grant, R. Abid, S. Collins, T. Martin, and T. Coles. In addition, we thank R.C. Reulen for his substantial help with data management and the West Midlands Cancer 
Intelligence Unit for providing data on patients with bladder cancer in the West Midlands. BCPP is supported by Cancer Research UK (grant number: CRUK/05/28).

Author contributions FHM van Osch: manuscript writing/editing, data analysis. D Nekeman: manuscript writing/editing, data analysis, data management. NK Aaronson: data collection, data analysis. LJ Billingham: project development, data analysis. ND James: data collection, project development. KK Cheng: project development. RT Bryan: project development, data management. MP Zeegers: project development.

\section{Compliance with ethical standards}

Conflict of interest The authors declare no potential conflicts of interest. All participants in this study provided written informed consent.

Open Access This article is distributed under the terms of the Creative Commons Attribution 4.0 International License (http://creativeco mmons.org/licenses/by/4.0/), which permits unrestricted use, distribution, and reproduction in any medium, provided you give appropriate credit to the original author(s) and the source, provide a link to the Creative Commons license, and indicate if changes were made.

\section{References}

1. Antoni S, Ferlay J, Soerjomataram I, Znaor A, Jemal A, Bray F (2017) Bladder cancer incidence and mortality: a global overview and recent trends. Eur Urol 71(1):96-108

2. Bryan RT, Zeegers MP, van Roekel EH, Bird D, Grant MR, Dunn JA et al (20131) A comparison of patient and tumour characteristics in two UK bladder cancer cohorts separated by 20 years. BJU Int 112(2):169-175

3. Boustead GB, Fowler S, Swamy R, Kocklebergh R, Hounsome L (2014) Section of oncology, BAUS. Stage, grade and pathological characteristics of bladder cancer in the UK: British association of urological surgeons (BAUS) Urol Tumour Registry. BJU Int 113(6):924-930.

4. van Rhijn BWG, Burger M, Lotan Y, Solsona E, Stief CG, Sylvester RJ et al (2009) Recurrence and progression of disease in non-muscle-invasive bladder cancer: from epidemiology to treatment strategy. Eur Urol 56(3):430-442

5. Monteiro LL, Witjes JA, Agarwal PK, Anderson CB, Bivalacqua TJ, Bochnerm et al. (2019) ICUD-SIU international consultation on bladder cancer 2017: management of non-muscle invasive bladder cancer. World J Urol 37:51-60

6. Sylvester RJ, van der Meijden APM, Oosterlinck W, Witjes JA, Bouffioux C, Denis L et al (2006) Predicting recurrence and progression in individual patients with stage Ta $\mathrm{T} 1$ bladder cancer using EORTC risk tables: a combined analysis of 2596 patients from seven EORTC trials. Eur Urol 49(3):466-477

7. Kamat AM, Hahn NM, Efstathiou JA, Lerner SP, Malmström P-U, Choi W et al (2016) Bladder cancer. Lancet 388(10061):2796-2810

8. Mowatt G, Zhu S, Kilonzo M, Boachie C, Fraser C, Griffiths TRL, et al (2010) Systematic review of the clinical effectiveness and cost-effectiveness of photodynamic diagnosis and urine biomarkers (FISH, ImmunoCyt, NMP22) and cytology for the detection and follow-up of bladder cancer. Health technology assessment, (Internet)

9. Svatek RS, Hollenbeck BK, Holmäng S, Lee R, Kim SP, Stenzl A et al (2014) The economics of bladder cancer: costs and considerations of caring for this disease. Eur Urol 66(2):253-262
10. Bryan RT, Kirby R, O’Brien T, Mostafid H (2014) So much cost, such little progress. Eur Urol 66(2):263-264

11. Van Der Aa MNM, Steyerberg EW, Sen EF, Zwarthoff EC, Kirkels WJ, Van Der Kwast TH et al (2008) Patients' perceived burden of cystoscopic and urinary surveillance of bladder cancer: a randomized comparison. BJU Int 101:1106-1110

12. Burchardt M, Burchardt, Shabsigh A, De La Taille A, Benson MC, Sawczuk I (2000) Current concepts in biomarker technology for bladder cancers. Clin Chem 46(5):595-605 (Internet).

13. Lotan Y, Roehrborn CG (2003) Sensitivity and specificity of commonly available bladder tumor markers versus cytology: Results of a comprehensive literature review and meta-analyses. Urology 61:109-118

14. Kirkali Z, Chan T, Manoharan M, Algaba F, Busch C, Cheng L, et al. (2005) Bladder cancer: epidemiology, staging and grading, and diagnosis. Urology 66:4-34.

15. D'Costa JJ, Goldsmith JC, Wilson JS, Bryan RT, Ward DG (2016) A systematic review of the diagnostic and prognostic value of urinary protein biomarkers in urothelial bladder cancer. Bl Cancer 2(3):301-317

16. Ward DG, Bryan RT (2017) Liquid biopsies for bladder cancer. Transl Androl Urol 6(2):331-335

17. Soria F, Droller MJ, Lotan Y, Gontero P, D'Andrea D, Gust KM et al (2018) An up-to-date catalog of available urinary biomarkers for the surveillance of non-muscle invasive bladder cancer. World J Urol 21:1-15

18. Bryan RT, Wei W, Shimwell NJ, Collins SI, Hussain SA, Billingham LJ, et al. Assessment of high-throughput high-resolution MALDI-TOF-MS of urinary peptides for the detection of muscle-invasive bladder cancer. Proteomics Clin Appl 2011; ;5:493-503

19. Zheng C, Lv Y, Zhong Q, Wang R, Jiang Q (2012) Narrow band imaging diagnosis of bladder cancer: systematic review and meta-analysis. BJU Int 110(11b):E680-E687

20. Vriesema JLJ, Poucki MH, Kiemeney LALM, Witjes JA (2000) Patient opinion of urinary tests versus flexible urethrocystoscopy in follow-up examination for superficial bladder cancer: a utility analysis. Urology. 56:793-7

21. Yossepowitch O, Herr HW, Donat SM (2007) Use of urinary biomarkers for bladder cancer surveillance: patient perspectives. J Urol 177:1277-1278

22. Zeegers MP, Bryan RT, Langford C, Billingham L, Murray P, Deshmukh NS et al (2010) The west midlands bladder cancer prognosis programme: rationale and design. BJU Int 105(6):784-788

23. Burger M, Catto JWFF, Dalbagni G, Grossman HB, Herr H, Karakiewicz P et al (2013) Epidemiology and risk factors of urothelial bladder cancer. Eur Urol 63(2):234-241

24. Aaronson NK, Ahmedzai S, Bergman B, Bullinger M, Cull A, Duez NJ et al (1993) The European organization for research and treatment of cancer QLQ-C30: a quality-of-life instrument for use in international clinical trials in oncology. J Natl Cancer Inst 85:365-376

25. Broadhead WE, Gehlbach SH, de Gruy FV, Kaplan BH (1988) The duke-UNC functional social support questionnaire. Measurement of social support in family medicine patients. Med Care 26(7):709-723

26. Von Neumann J, Morgenstern O (1944) Theory of games and economic behavior. Princet Univ Press

27. Cauberg ECC, de Bruin DM, Faber DJ, van Leeuwen TG, de la Rosette JJMCH, de Reijke TM (2009) A new generation of optical diagnostics for bladder cancer: technology, diagnostic accuracy, and future applications. Euro Urol 56:287-297 
28. Bryan RT, Billingham LJ, Wallace DMA (2008) Narrow-band imaging flexible cystoscopy in the detection of recurrent urothelial cancer of the bladder. BJU Int 101:702-716

29. James ND, Hussain SA, Hall E, Jenkins P, Tremlett J, Rawlings C et al (2012) Radiotherapy with or without chemotherapy in muscle-invasive bladder cancer. N Engl J Med 366(16):1477-1488
Publisher's Note Springer Nature remains neutral with regard to jurisdictional claims in published maps and institutional affiliations. 